should aim at making a large coloboma, extending quite up to the periphery of the iris. I have seen only one accident produced by iridectomy, and that was in a hospital patient, in whom the adhesions were so firm as to tear the anterior capsule when the iris was drawn out of the eye, and so produce traumatic cataract. The lens was removed shortly afterwards, and the case altimately did well, the eye recovering useful vision.

A case of very unusual character, which in some respects combined the characters of ordinary plastic and of serous iritis, was that of a girl twelve years of age, who was brought to me in October, 1891, with severe iritis of the right eye, which was said to have originated in scarlet fever in the preceding January, and which had been recurrent on more than one occasion. The pupil was contracted and there was no measurable vision. Atropine and perchloride of mercury were prescribed, and in a fortnight the patient returned with the active inflammation subdued and the pupil generally dilated, but with an adhesion on the nasal side and with much deep opacity. The vision was then th the normal and the treatment was left in the hands of the family medical attendant. In March, 1892, I found the adhesion detached and the vision increased to 1 th ; but there were numerous flocculi in the vitreous and the surface of the optic disc was uniformly red, so as only to be distinguishable from the rest of the fundus as the meeting point of the retinal vessels. Cautious use was permitted; and in the following November the vision of the right eye remained stationary, but the vitreous was less cloudy. The vision of the left eye, which had previously been normal, had begun to fail, and was only $\frac{2}{5}$ ths of the normal, whilst the left vitreous contained flocculi, and the left disc was as red as the right. Since that time the patient has remained under $m y$ observation and has taken perchloride of mercury constantly. She is in excellent bodily health, and has gradually brought her eyes more and more into use for educational purposes. She suffers no pain or discomfort, and is at present allowed to read for an hour twice a day. The vision of her right eye has for some months been stationary at $\frac{1}{2}$, and that of the left eye at about $\frac{2}{3} \mathrm{rds}$; but the vitreous of both eyes is full of particles, and both dises are as red, or nearly as red, as ever. It is barely possible to perceive their outlines. For a long time I was apprehensive of the existence of a chronic neuritis which might ultimately bring about atrophy by the contraction of effused material; but the unchanging condition of affairs now leads me to hope that there is nothing more than a capillary hyperæmia, left behind by the original malady. It is not a little remarkable that the left eye has never suffered from iritis, although the deep changes of which it has been the seat have been precisely like those which followed iritis in the right eye. I have more than once seen instances of lost or greatly impaired vision from complete or partial nerve atrophy which was said to have originated in scarlet fever; but I have not met with any other case of iritis which appeared to be connected with scarlet fever, or of double nerve congestion of which iritis of one eye only had been the precursor.

Amongst cases of iritis distinguished from others only by their great severity mention should be made of those, unfortunately not as rare as they should be, in which the disease had been aggravated in its early stage by the application of some form of irritant, such as a solution of a salt of lead or of zinc. Such applications are usually the result of domestic medication, but $I$ have known them to be prescribed by practitioners who failed to recognise the true nature of the affection, and who mistook impending iritis for a simple inflammation of the conjunctiva. An application from which $I$ have seen much injury is vinum opii, the amount of opium in a drop being too insignificant to exert any sedative effect, whilst the alcoholic menstruum is an irritant of the most pronounced character. The general principle, when in doubt to use atropine, is one which cannot be too strongly impressed upon the mind of every person to whom an inflamed eye may be brought for treatment. It is possible, nevertheless, to commit the converse error. I have lately seen a gentleman advanced in life, in one of whose eyes a single adhesion had been left behind by an attack of iritis from which he had suffered thirty years before. The adhesion had been so stretched that it did not restrain pupillary movement, and was harmless. A few weeks since he had a trivial conjunctivitis of both eyes ; and the adhesion was not only discovered, but was held to justify an active treatment by atropine, mercury, and functional rest, the grounds of which were not at first disclosed to the patient.
When he was made acquainted with them, he not unnaturally found some solace in resorting to the advice of a different medical attendant. In non-syphilitic cases associated with a lithatic diathesis, and marked by a strong tendency to recurrence, a great deal may often be done to obviate this tendency by means of careful regulation of the diet and habits of life. The sparing use, or even the complete abandonment, of animal food, whilst the action of the skin and of the kidneys is encouraged by exercise, clothing and diluents, and that of the liver is stimulated by an occasional mild mercurial, forms the outline of a plan which must be varied to meet the requirements of individual cases, but which I have often pursued with great advantage. I have seen instances in which what seemed to be an established habit of recurrence was at last effectually broken through by perseverance on these lines, the patient being instructed to watch his urine daily, and to regard the appearance of deposited lithates as a storm-warning which he was by no means to neglect. When adhesions have been left behind by a former attack or attacks of iritis, and recurrence is either threatened or has occurred, the question of how these adhesions should be dealt with assumes considerable practical importance. It has always seemed to me that the effect of one or two adhesions of limited extent is far more injurious, regarded as causes of recurrence, than the effect of adhesions of a more extensive character, by which the muscles of the iris are more effectually restrained, and against which they cease to strive. The latter, indeed, point to risks of a different character, because they bring complete occlusion of the pupil into view as a near probability, but they are less frequently the exciting causes of the inflammation by which such occlusion may be produced. For adhesions of small extent the methods of detachment, contrived respectively by the late Mr. Streatfeild and by Dr. Passavant, would naturally suggest themselves as preferable to the disfigurement of an iridectomy; but the suggestion does not appear to me to be supported by practical experience. In either of these methods, whether the adhesion be detached by traction towards the pupil or towards the periphery, there is equally a danger that the capsule of the lens may tear more readily than the material of the adhesion and that a traumatic cataract may be produced; whilst there is also the danger of producing a concealed adhesion by the operation itself-an adhesion, that is, between the peripheral part of the iris and the cicatrix of the operation wound. Both these risks are set aside by iridectomy, which may always be so planned as to include only the non-adherent portions of the iris, and which, when done in this way, seems, by the destruction of the continuity of the muscular circle, to be completely efficacious in overcoming the irritation which the arrest of movement by adhesions is otherwise calculated to produce.

(To be continued.)

\section{EXPERIMENTS WITH CHLOROFORM AND} ETHER : ${ }^{1}$

CONDUCTED AT HYDERABAD, DECCAN.

BY C. H. LEAF, M.B. CANTAB.

THE following is an account of a series of experiments which were performed in the Laboratory of the Hyderabad Medical School, with the permission of Surgeon-Lieutenant-Colonel E. Lawrie, by Messrs. Leaf, Kelly and Chamarette, with the help of students Mahomed Abdul Gani, Ismael Khan and Abdul Hafiz. They were conducted with the view of watching the effects of chloroform on the respiration and circulation, and were for the most part extremely simple. The method employed con sisted in first placing pariah dogs into a wooden box $3 \mathrm{ft} .10 \mathrm{in}$. long by $1 \mathrm{ft}$. $5 \mathrm{in}$. broad and $1 \mathrm{ft}$. $7 \mathrm{in}$. deep. The lid had an opening through which pieces of blotting-paper saturated with chloroform were passed. The movements of the animal were watched through glass placed over the opening. As soon as insensibility was produced and the dog had fallen down it was removed on to a table. Chloroform was then administered to it on a cloth cap at the top of which a little cotton-wool

1 This paper was accompanied by a volume of over 200 quarto pages containing details of the experiments. 
was stitched. The respiratory movements were carefully watched and the action of the heart (except in a few cases where a portion of the thoracic wall was removed) was observed by the vibrations of a needle thrust into the organ through the chest wall.

One hundred and sixty-four experiments were performed -one hundred and thirty on dogs and thirty-four on frogs. Before describing the experiments we may mention that in mo instance, excepting one-viz., Experiment 10-was a dog anintentionally killed by an overdose. In this case, as the animal was very savage, and as it was removed from the box too soon, chloroform had to be vigorously pushed during the struggling stage.

The experiments were conducted with the following objects: (a) To observe the effects on the respiration and circulation of chloroform administered in different ways; (b) to test the value of the different means of restoring animals after the respiration had stopped; $(c)$ to observe the effects of temporary stoppage of the heart, whether naturally or artificially produced.

The following experiments were therefore made: (a) Stimulation of the vagus under chloroform, $(b)$ administration of enormous doses of chloroform before and after section "of vagi, (o) administration of chloroform whilst the wreathing was irregular and the animal struggling, $(d)$ artificial respiration with and without bellows, $(e)$ inflation of the lungs with oxygen, and $(f)$ injections of ether and strychnine. ${ }^{2}$

Stimulation of the vagus after the respiration ceased, which was tried in numerous cases, showed that the effects produced on the heart were not uniform. (By " respiration ceased" is meant not merely the non-entrance of air into the chest, but the entire absence of all abdominal and thoracic movements.) In some cases no apparent effect was produced on the needle movements ; in others the movements were much quickened, but in the majority of cases they were markedly slowed or completely stopped for a longer or shorter period. In twenty-five dogs in which stimulation of the vagus was performed on the first occasion the respiration stopped; eleven recovered and fourteen died. Of the latter there were seven cases in which no slowing effect was produced on the heart and there were only three cases in which stimulation was commenced immediately the respiration ceased. In the eleven that recovered there were two cases in which it was doubtful whether any effect was produced, and two cases only in which stimulation was commenced immediately the respiration ceased. Experiments $8,9,12 \mathrm{~A}, 13,16 \mathrm{~A}, \mathrm{~B}, \mathrm{C}$, and $\mathrm{D}, 18 \mathrm{~B}$ and $\mathrm{C}, 24$, $26,28,59,72,107$ and 120 show that the stoppage or slowing of the heart which was artificially produced after the cessation of respiration (and which occurred in all but two cases) in no way interfered with, if it did not actually aid in, the animal's recovery. It should also be noted that the stimulation of the vagus was not commenced in every case immediately, but was delayed on an average twenty or thirty seconds after the respiration had ceased. It was found that stimulation during normal anæsthesia did not interfere with the dog's subsequent recovery. These facts show that the temporary stoppage or slowing of the heart, which was almost invariably produced by the stimulation of the vagus whether occurring before or after the cessation of respiration, is per se harmless. In support of this truth, which was discovered by the Hyderabad Chloroform Commission, Experiment 50 may be adduced. In this experiment the heart stopped beating for fifty seconds between the cessation of breathing and the commencement of artificial respiration and the dog recovered. When more chloroform was subsequently given till the respiration ceased it again recovered. This shows conslusively that the fact of the heart having stopped is no proof of its weakness, and as we have never found the heart stop even temporarily during anæsthesia, or simultaneously with the cessation of the respiration, in those cases in which both vagi have been divided, whereas it is common in dogs whose vagi are intact, we are led to believe that the stoppage which occurred in this case was purely reflex in origin. With the view of observing whether the heart's action could, apart from reflex stoppage, be arrested temporarily or be affected in any way prior to respiratory failure, we performed several experiments, in which both vagi were divided and the effects

2 The current from an ordinary induction coil was always employed in these experiments and tested berore being used, but it was frequently out of order and always uncertain. of administering enormous doses of chloroform were noted Experiments 71 and 116, also 109, are good examples. In these cases enormous doses of chloroform were given, but at no time prior to respiratory embarrassment was any feebleness of the needle movements observed. The administration of the largest possible doses of chloroform produced no prejudicial effect on the heart, and it is certain that no amount of chloroform given by inhalation can produce cardiac syncope or any direct effect on the heart until the respiration ceases. The following facts prove this : (1) The heart needle continued beating vigorously for a considerable time after the respiration had ceased; (2) both animals twice recovered spontaneously after the cessation of respiration. Moreover, when the respiration finally stopped for a fourth time in Experiment 116 the needle was still beating vigorously. In dogs, therefore, it is abundantly evident that the heart beats effectively after stoppage of the respiration by an overdose of chloroform and that temporary stoppage of the heart, whether produced spontaneously or artificially, is not a danger but a safeguard. This fact was originally discovered by Dr. Bomford and is strongly insisted upon by the Hyderabad Commission.

We found that when chloroform was given to an animal whilst it was struggling violently and holding its breath the needle frequently stopped for some seconds, either during this period or very shortly afterwards. This was of such frequent occurrence that at first it seemed as though the needle, owing to the violent struggles, bad been displaced; but that this was not so is proved by the fact that the needle in the majority of cases, without being removed and reinserted, continued beating regularly later. In Experiment 32 the needle stopped completely for 2 min. 10 sec. during the struggling stage.

In Experiment 116 chloroform was given whilst the animal struggled violently. The effect of this on the needle beats was to make them extremely intermittent and irregular, not only during the struggling period, but more or less throughout the whole Observation A. When, subsequently, both vagi were divided the needle beats at once became regular, showing that the intermittency and irregularity, which were noticed in the first Observation A, could not have been produced by the direct action of chloroform on the heart, but were entirely reflex in orgin.

In Experiment 116 the needle beats were more vigorous in Observation D after the section of the vagi than they were in Observation $A$, when the vagi were intact, the respiration having stopped in both cases. The irregularity and intermittency of the needle which were noticed in Observation A were undoubtedly due to the fact that chloroform was given continuously whilst the animal struggled violently and the respiration was irregular. Cne effect therefore of giving chloroform during the struggling stage, or when the breathing is irregular, is to cause inhibition or extreme intermittency of the heart which may last throughout the whole administration, although the breathing may subsequently become regular. We found that after administering chloroform in this way the respiration would frequently stop very suddenly and unexpectedly ; especially was this the case in large, strong, healthy pariahs. ${ }^{3}$ In all those cases that we failed to revive by pumping in air with bellows one minute after the respiration ceased, chloroform was thus administered with the cap tightly jammed over the face, and artificial respiration was far less successful in restoring these animals than, judging from their size and strength, we should have imagined would have been the case.

There was also another period during which the heart needle would stop temporarily, and this was either a few seconds before, simultaneously with, or immediately after the stoppage of the respiration. These temporary stoppages, which were fairly common, as already mentioned, were undoubtedly due to inhibition of the heart through the vagi, for when these nerves were cut they never occurred.

After the temporary stoppages we found the needle would always go on beating for some time, generally some minutes, after the respiration had ceased. In no single case did the needle ever stop permanently at the same time as the respiration. It was a common occurrence to find the needle beating fairly vigorously, though no pulse could be felt, as in Experiments 13 and 16 . The stoppage of the heart needle movements therefore is notnecessarily an indication that the heart has failed or is weakened; it may be and frequently

3 In the only accidental death that occurred chloroform was pushed during the struggling stage. 
is a sign of activity of the cardio-inbibitory centre, for we frequently found, when chloroform was pushed on two or more occasions until the respiration ceased, that the heart was much more likely to be inhibited on the first than on the last occasion, and so far from the needle beats becoming less vigorous after increasing doses of chloroform the exact contrary was observed, showing that the temporary stoppage and apparent weakness of the heart's action were brought about by the activity of the cardio-inhibitory centre, which on the later occasions became paralysed, the result being that the beats of the heart became rapid and vigorous and the effect produced was exactly the same as though both vagi had been cut.

In the experiments with artificial respiration dogs were anæsthetised as before until the respiration ceased, and then after varying intervals of time an attempt was made to revive them by pumping in air by means of an ordinary pair of bellows, the nozzle of which passed into an opening made in the trachea. After each inflation pressure was made on the thorax so as to imitate as far as possible natural respiration. Of fourteen dogs that we experimented on in this way, commencing artificial respiration one minute after all movements of the chest and abdomen ceased, ten recovered and four did not. In all the cases chloroform was vigorously pushed with the cap tightly pressed over the face in the struggling stage. We found that artificial respiration with bellows was sometimes successful in restoring an animal some considerable time after the respiration had ceased. In Experiment 41 the animal was twice restored, the first time 3 min. 20 sec. and the second time $6 \mathrm{~min}$. $25 \mathrm{sec}$. after the respiration had ceased, notwithstanding the fact that in the latter case a large dose of chloroform had been given when the dog was struggling violently. Again, in Experiment 40 a very weak dog was three times resuscitated by artificial respiration, on the last occasion $5 \mathrm{~min}$. $10 \mathrm{sec}$. after the respiration had ceased. These experiments show that dogs could often be restored some considerable time after the respiration had ceased and that by performing artificial respiration with bellows we found we were able to revive them after a longer interval than was possible by the ordinary manual method, where the longest interval which elapsed was $4 \mathrm{~min}$. 40 sec.

Acting on the suggestion of Mr. Foy, experiments were made with a view to testing the value of oxygen gas in cases of chloroform poisoning. The gas was made by mixing dioxide of manganese and chlorate of potassium in a glass retort which was heated by a spirit lamp. As the gas was generated it was purified by bubbling through water contained in a gas-holder capable of containing 1500 cubic inches. When the receiver was full it was connected by a tube to a pair of bellows, the nozzle of which fitted into an opening made in the trachea. The gas was forced ont by pressure of water from above. In this way the oxygen contained in the receiver could be pumped directly into the lungs. After each inflation with the gas pressure was made on the thorax so as to imitate as far as possible natural respiration. The amount of gas used on each occasion varied from 100 to 500 cubic inches. Nine experiments were performed in this manner, and we found that we could only revive three of the nine dogs by artificial respiration with oxygen one minute after the respiration had ceased. Except in Experiment 100 , in which a small puppy was twice revived one minute after the respiration had ceased, there was nothing to show that oxygen gas, apart from the expansion of the lungs which was necessarily produced at the same time, had any intrinsic effect in restoring the animal to life. We were never able in these experiments to restore a dog after a longer interval than one minute. Oxygen is therefore clearly not an antidote in chloroform poisoning.

Two experiments were made with injections of strychnine. In the first $\frac{1}{2}$ th of a grain was injected forty seconds and in the second $\frac{1}{5}$ th of a grain thirty seconds after the respiration had ceased, but no recovery followed in either case.

Twelve experiments were made with a view of watching the effects of subcutaneous injections of thirty minims of ether after the respiration had ceased. We found that of twelve dogs only four recovered, and of these four artificial respiration was practised in three. In other words, only one dog recovered after the injection of ether alone. We frequently noticed that after the injections the needle movements were temporarily quickened, but subsequently stopped abruptly. In some cases injections of ether produced no obvious alteration in the needle beats. There was nothing to show that injections of ether produced the slightest effect in restoring the animal. On the other hand, the fact of there being only one recovery and of the needle stopping abruptly undoubtedly pointed to an injurious effect. It is obvious that injections of ether are useless, if not positively harmful, in overdosing with chloroform.

Experiments 121 to 124 show the effect of injections of chloroform into the cavities of the ventricles. Experiments 125 and 126 show the effect of these injections into the ventricular substance. In both series weakness and intermittency of the heart's action were produced, but not sudden stoppage. Experiment 134 shows that the final effect of injecting ether into the substance of the heart is exactly the same as that of chloroform. Experiments 131 and 133 show the effects of filling the pericardium with chloroform and ether respectively; no visible prejudicial effect on the movements of the heart was observed in Experiment 131 until artificial respiration was stopped, and though the pericardium was filled with chloroform the heart continued to beat vigorously for twenty-one minutes. Experiment 135 shows the effect on the heart of blowing in concentrated chloroform. ${ }^{4}$ In this case each time concentrated chloroform was pumped in by the bellows the veins on the surface of the ventricle became prominent, the ventricular muscle became relaxed, and the beats had more resemblance to a flap than a true contraction.

The experiments on frogs' hearts show that both auricles and ventricles will go on beating for a ccnsiderable time whether the heart is soaked in liquid chloroform on exposed to its vapour. The only occasions on which the hearts were found to be quite motionless on opening the thorax were in Experiments 156 and 162. Here the hearts were found to be enormously distended, showing that they had failed through the respiration.

\section{General Conclusions.}

Our experiments prove what Bernard has shown and what Dr. Lauder Brunton and others have long insisted upon-viz., that chloroform is an irritant and kills protoplasm. Frogs' hearts hung up in the vapour of chloroform or immersed in the liquid ceased to beat, and the cessation ther took place more rapidly than when they were suspended in air or immersed in water. When chloroform was injected into the heart substance of dogs it irritated and probably coagulated the protoplasm and the organ was rapidly brought to a standstill. Precisely the same effects, only less intense, were produced with ether, and similar effects, only much more intense, were produced with dilute hydrochloric acid. Many irritants act like chloroform. The peculiarity of chloroform is that its undiluted vapous seems to be irritating in much the same way as the fiuid, which is not the case with other irritating substances, such as alcohol or dilute hydrochloric acid. The irritating and coagulating action cannot occur in the ordinary use of chloroform, and there is no more fear of coagulation of the heart than there is of coagulation of the general muscles, or of sloughing of the toes or stomach, or of necrosis of the tissues generally - all of which may be produced by chloro. form locally applied.

The entire series of experiments tends to show that, although hearts placed in abnormal conditions may be weakened and stopped by overdoses of chloroform, under no circumstances can sudden cardiac failure and death from reflex stoppage take place; nor, up to the cessation of respiration, can any direct effect be produced upon the heart by chloroform unless it be injected into its substance or inta its cavities. In those experiments where both vagi had been divided no obvious effect could be produced on the heart beats by chloroform, administered in the largest possible doses by inhalation, before failure of the respiration; and finally, in the experiments in which the thorax was opened and artificial respiration was necessary throughout, if this was discontinued even for a brief interval, the action of the heart was quickly arrested. It is therefore clear that no amount of chloroform given by inhalation can act prejudicially and directly on the heart muscle before interfering with the breathing, and in practice the remote possibility of 4 Two experiments were subsequently made in which chlorine was
blown into the lungs, and in both cases the contractions rapidly became feeble and ineffective. The right ventricle remained full, though not distended. When the chlorine was discontinued the beats regtined their strength, and vice versa. In these two cases and in the case of chloroform the results were probably caused by interference with the respiratory function. 
danger arising in this way is so infinitesimal that it may be entirely disregarded.

Our experiments lay no claim to originality, but as far as they go they altogether confirm the results arrived at by the Hyderabad Chloroform Commissions.

Upper Gloucester-place, Baker-street, W.

$=\frac{\text { CASE OF }}{\text { SPONDYLOLISTHESIS ASSOCIATED WITH }}$
PROGRESSIVE PARAPLEGIA;
LAMINECTOMY.

BI W. ARBUTHNOT LANE, M.S. LOND., F.R.C.S. ENG. ASSISTANT SURGEON TO GUY'S HOSPITAL AND TO THE HOSPITAL FOR SICK CHILDREN, GREAT ORMOND-STREET.

A WOMAN aged thirty-five was admitted into Guy's Hospital ander my care on March 24th, 1893 . She stated that she had always been a hard-working woman, performing usually the duties of a general servant. Twelve years ago she had a master who was a lunatic and whose delusion consisted in believing that he was always playing cricket. He used to go about with a very heavy stick and struck at articles of furniture imagining them to be cricket balls. On several occasions he had come behind her unexpectedly and had struck her violently in the lower part of the back with this stick. She had suffered great pain from the blows, and, as far as she could remember, she localised the parts struck as the lower part of the lumbar spine and the buttock. She said that the blows that were struck with the heavy stick were so severe that if she had not been supporting herself she would kave been knocked down. Up to this time and for about five years later she had been a strong robust woman and had felt no permanent effect from the injuries received, though she seemed inclined to ascribe her present condition to them. As far as she was aware her attitude and gait differed in no way from those of other women. About six years ago she noticed that her walk was insecure and that she rolled about, especially to the right side. Her back also became weak, and though she suffered no pain there she found a difficulty in carrying heary things up and down stairs especially. This weakness of her back and insecurity on her legs increased steadily, and she found that the right leg was much less reliable than the left. A deformity of the lumbar spine had also been observed during the last nine months. About a year ago she was no longer able to walk. Six months ago she was admitted to the Camberwell Infirmary, where she remained till her admission into Guy's Hospital. Recently the right foot had become extended and the toes flexed. She had found that sensation had steadily diminished in the lower extremities. Although I have questioned her most closely I cannot obtain any evidence of deformity or peculiarity in gait prior to six years ago. She had never suffered from pain in her limbs or in her back.

On admission the patient looked older than her age. The form of the lumbar spine was altered, being somewhat convex posteriorly, the prominence of the convexity corresponding to apices of the spinous processes of the third and fourth lumbar vertebræ. These seemed on an abnormally low level as compared with the iliac crests. The spine of the fifth lumbar rertebra could be felt only with difficulty, and seemed to be unusually deeply buried. Sensation of touch, of heat, of pain and of cold was impaired to some extent over almost the whole of both lower extremities, though in parts it was very slightly affected. This was much more marked in some places, as, for instance, below the right knee and especially in the foot, and again in parts of the buttock and about the anus. It was rery difficult to define with any accuracy the limits of the more or less insensitive areas and the degree of the loss of sensation. It was certainly very much more deficient on the right than on the left side. There was slight cedema of both legs below the knees and especially of the right. The circumference of the calf of the right leg measured $10 \frac{1}{4}$ in., and that of the left $11 \frac{1}{4}$ in. The right foot was extended and the toes were somewhat flexed. It was not possible to overcome this attitude completely by manipulation. The patient was able to flex and extend the hip-joint and the knee-joint with a considerable amount of power. The force that could be exerted spontaneously over the foot and ankle was fair, though subnormal, on the left side, but was practically nil on the right. The knee-jerks were completely absent on both sides, and it was not possible to obtain any ankle clonus. A plantar reflex was obtained, but of an indefinite character. It was obvious that the cauda equina was compressed, and compressed very unequally. Beyond the fact of the spinous process of the fifth lumbar being apparently somewhat deeply placed there was nothing to suggest anything abnormal in the lumbo-sacral joint. This was rendered also less definite by the fact of the spinous process being abnormally developed even for a woman. ${ }^{1}$ Though there was much evidence, both in the history of the case and in the condition of the patient, to contraindicate compression paraplegia, due to spinal caries, I came to the conclusion that this was the most probable cause. In any case an operation seemed advisable. Consequently, on March 28th I removed the spine and lamina of the fourth lumbar vertebra, when it was quite obvious that the compression was below this level, because the dura matral sheath was distended with fluid. From the great density of the spinous process and lamina $I$ concluded at once that there was no carious focus in the vicinity. On attempting to remove the lamina of the fifth lumbar vertebra, after cutting off its very prominent, largely developed and dense spinous process, it was found to be placed in the upper part of the sacral canal quite in front of its normal position. It was removed piecemeal with great difficulty, when the dura matral sheath of the cauda equina on the right side was seen to have been so severely compressed as not to expand when the bone pressing on it had been removed, nor was it possible to separate the opposing dura matral surfaces with dissecting forceps. The left half filled up freely at once with cerebro-spinal fluid when the segment of lamina compressing it was cut away. The fifth lumbar nerve on the right side seemed to be com pressed between the lamina and the body of the sacrum, but I was not sure whether the corresponding nerve on the left side had sustained any pressure or not. The superior and posterior margin of the body of the first piece of the sacrum stood out prominently and sharply, and the posterior surface of the body of the fifth lumbar vertebra could be felt about threequarters of an inch in front of its normal position. There was, as far as could be seen, no solution of continuity of the neural arch at any point on either side, though on both sides it was deeply channelled by the articular processes of the fourth lumbar and first sacral vertebræ. At the same time, at such an operation it was impossible to follow out details with the same accuracy that a dissection allows of. No evidence of any fracture was found. Beyond the excessive amount of forward displacement the conditions discovered differed in no manner from the cases of spondylolisthesis which I have dissected and described. The wound was closed and a drainage-tube was left in for twenty-four hours. This was removed and the aperture was accurately closed with a suture, primary union resulting. It was obvious that the alteration in the character of the lumbar curve was consequent upon the forward displacement of the fifth lumbar vertebra, and was brought about mechanically to obviate the slipping forwards of the vertebra by a displacement backwards of the centre of gravity of the superjacent trunk.

This case is obviously of great interest from its rarity. I am not aware of any reported case of spondylolisthesis of the fifth lumbar vertebra in which pressure on the lumbar or sacral nerves was described. When working at the changes which the skeletons of labourers undergo I found that in fully developed coal heavers spondylolisthesis is the normal condition and that in other occupations a similar displacement existed. The character of the displacement varied in different individuals with the particular mode of transmission of pressure. In the coal heaver, ${ }^{2}$ for instance, the displaced body of the fifth lumbar vertebra was almost completely ankylosed to the sacrum, the fibro-cartilage being practically absent, whilst in other occupations ${ }^{3}$ the $\cdot$ spondylolisthetic vertebra formed an arthrodial joint with the sacrum. In other occupations, again, I showed that backward displacement of the body of the fifth lumbar ${ }^{4}$ occurred normally-a condition which had not been previously described or observed.

1 I have descrihed in a paper in the Transactions of the Obstetrical Society, 1887 (the Factors which Determine the Variations in the Form of the Male and Female Pelvis), and in other papers the different sexes and the consequent difference in the anatomy of these bones and joint.

${ }^{2}$ See Fig. 3, Plate XIII., in the Transactions of the Pathological Society, 1885 3 See Fig. 2, Plate XIII., in the Transactions of the Pathological
Society, 1885.

4 See Fig. 1 of the same plate. 